\title{
Comet 67P/Churyumov-Gerasimenko: Constraints on its origin from OSIRIS observations
}

\author{
H. Rickman ${ }^{1,2}$, S. Marchi ${ }^{3}$, M. F. A'Hearn ${ }^{4}$ C. Barbieri ${ }^{5}$, M. R. El-Maarry ${ }^{6}$, C. Güttler ${ }^{7}$, W.-H. Ip ${ }^{8}$, H. U. Keller ${ }^{9}$,
} P. Lamy ${ }^{10}$, F. Marzari ${ }^{5}$, M. Massironi ${ }^{11,12}$, G. Naletto ${ }^{12,13,14}$, M. Pajola ${ }^{12}$, H. Sierks ${ }^{7}$, D. Koschny ${ }^{15}$, R. Rodrigo ${ }^{16,17}$, M. A. Barucci ${ }^{18}$, J.-L. Bertaux ${ }^{19}$, I. Bertini ${ }^{12}$, G. Cremonese ${ }^{20}$, V. Da Deppo ${ }^{14}$, S. Debei ${ }^{21}$, M. De Cecco ${ }^{22}$, S. Fornasier ${ }^{18}$, M. Fulle ${ }^{23}$, O. Groussin ${ }^{10}$, P. J. Gutiérrez ${ }^{24}$, S. F. Hviid ${ }^{25}$, L. Jorda ${ }^{10}$, J. Knollenberg ${ }^{25}$, J.-R. Kramm ${ }^{7}$, E. Kührt ${ }^{25}$, M. Küppers ${ }^{26}$, L. M. Lara ${ }^{24}$, M. Lazzarin ${ }^{5}$, J. J. Lopez Moreno' ${ }^{24}$, H. Michalik ${ }^{27}$, L. Sabau ${ }^{28}$, N. Thomas ${ }^{6}$, J.-B. Vincent ${ }^{7}$, and K.-P. Wenzel ${ }^{15}$

${ }^{1}$ P.A.S. Space Research Center, Bartycka 18A, 00-716 Warszawa, Poland

2 Dept. of Physics and Astronomy, Uppsala University, Box 516, 75120 Uppsala, Sweden

3 Southwest Research Institute, 1050 Walnut St., Boulder, CO 80302, USA

e-mail: marchi@boulder.swri.edu

4 Department for Astronomy, University of Maryland, College Park, MD 20742-2421, USA

5 Department of Physics and Astronomy "G. Galilei”, University of Padova, Vic. Osservatorio 3, 35122 Padova, Italy

${ }^{6}$ Physikalisches Institut, Sidlerstrasse 5, University of Bern, 3012 Bern, Switzerland

7 Max-Planck Institut für Sonnensystemforschung, Justus-von-Liebig-Weg 3, 37077 Göttingen, Germany

8 Institute for Space Science, National Central University, 32054 Chung-Li, Taiwan

9 Institute for Geophysics and Extraterrestrial Physics, TU Braunschweig, 38106 Braunschweig, Germany

10 Aix-Marseille Université, CNRS, LAM (Laboratoire d'Astrophysique de Marseille) UMR 7326, 13388 Marseille, France

11 Dipartimento di Geoscienze, University of Padova, via G. Gradenigo 6, 35131 Padova, Italy

12 Centro di Ateneo di Studi ed Attivitá Spaziali, "Giuseppe Colombo" (CISAS), University of Padova, via Venezia 15, 35131 Padova, Italy

13 Department of Information Engineering, University of Padova, via G. Gradenigo 6/B, 35131 Padova, Italy

14 CNR-IFN UOS Padova LUXOR, via Trasea 7, 35131 Padova, Italy

15 Scientific Support Office, European Space Agency, 2201 Noordwijk, The Netherlands

16 Centro de Astrobiologia (INTA-CSIC), 28691 Villanueva de la Canada, Madrid, Spain

17 International Space Science Institute, Hallerstrasse 6, 3012 Bern, Switzerland

18 LESIA, Obs. de Paris, CNRS, Univ. Paris 06, Univ. Paris-Diderot, 5 Pl. J. Janssen, 92195 Meudon, France

19 LATMOS, CNRS/UVSQ/IPSL, 11 bd d'Alembert, 78280 Guyancourt, France

20 INAF-Osservatorio Astronomico, vicolo dell'Osservatorio 2, 35122 Padova, Italy

21 Department of Mechanical Engineering, University of Padova, via Venezia 1, 35131 Padova, Italy

22 UNITN, Univ. di Trento, via Mesiano 77, 38100 Trento, Italy

23 INAF-Osservatorio Astronomico, via Tiepolo 11, 34143 Trieste, Italy

24 Instituto de Astrofisica de Andalucía (CSIC), Glorieta de la Astronomía s/n, 18008 Granada, Spain

25 Institute of Planetary Research, DLR, Rutherfordstrasse 2, 12489 Berlin, Germany

26 Operations Department, European Space Astronomy Centre/ESA, PO Box 78, 28691 Villanueva de la Canada, Madrid, Spain

27 Institut für Datentechnik und Kommunikationsnetze der TU Braunschweig, Hans-Sommer-Str. 66, 38106 Braunschweig, Germany

28 Instituto Nacional de Técnica Aeroespacial, Carretera de Ajalvir, p.k. 4, 28850 Torrejon de Ardoz, Madrid, Spain

Received 13 March 2015 / Accepted 12 May 2015

\section{ABSTRACT}

Context. One of the main aims of the ESA Rosetta mission is to study the origin of the solar system by exploring comet $67 \mathrm{P} /$ Churyumov-Gerasimenko at close range.

Aims. In this paper we discuss the origin and evolution of comet 67P/Churyumov-Gerasimenko in relation to that of comets in general and in the framework of current solar system formation models.

Methods. We use data from the OSIRIS scientific cameras as basic constraints. In particular, we discuss the overall bi-lobate shape and the presence of key geological features, such as layers and fractures. We also treat the problem of collisional evolution of comet nuclei by a particle-in-a-box calculation for an estimate of the probability of survival for 67P/Churyumov-Gerasimenko during the early epochs of the solar system.

Results. We argue that the two lobes of the 67P/Churyumov-Gerasimenko nucleus are derived from two distinct objects that have formed a contact binary via a gentle merger. The lobes are separate bodies, though sufficiently similar to have formed in the same environment. An estimate of the collisional rate in the primordial, trans-planetary disk shows that most comets of similar size to 67P/Churyumov-Gerasimenko are likely collisional fragments, although survival of primordial planetesimals cannot be excluded. Conclusions. A collisional origin of the contact binary is suggested, and the low bulk density of the aggregate and abundance of volatile species show that a very gentle merger must have occurred. We thus consider two main scenarios: the primordial accretion of planetesimals, and the re-accretion of fragments after an energetic impact onto a larger parent body. We point to the primordial signatures exhibited by 67P/Churyumov-Gerasimenko and other comet nuclei as critical tests of the collisional evolution.

Key words. comets: individual: 67P/Churyumov-Gerasimenko 


\section{Introduction}

Promoting the quest for the origin of the solar system is fundamental to the concept of the ESA/Rosetta mission. A comet nucleus - specifically, that of 67P/Churyumov-Gerasimenko (hereinafter 67P) - was chosen as the target because of the common notion that comet nuclei are icy planetesimals formed beyond the snow line in the nascent solar system and the evidence that their atomic and molecular compositions provide a good match to the primordial material out of which the solar system was built. In particular, comet 67P was selected as the back-up target after the failure to launch Rosetta toward 46P/Wirtanen, partly because it was expected to be relatively fresh after having recently encountered Jupiter (in 1959) and thus had its perihelion distance much reduced.

The formation of the icy planetesimals is currently viewed within either of two different scenarios. One is the classical model of hierarchical accretion (Weidenschilling 2008), and the other considers the assembly of gravitationally unstable "pebble clouds" by gas-grain instabilities in the solar nebula (Johansen et al. 2014). Recent simulations (Wahlberg Jansson \& Johansen 2014) have shown how planetesimals in the size range from 10 to $1000 \mathrm{~km}$ can thus be produced.

Independent of which scenario is relevant to comet 67P, it seems clear that its ultimate origin has to be sought in the same, very early environment. The exact place is more difficult to specify. The region inside the initial orbits of the giant planets can likely be discarded, since cometary ices would not condense there. Because of the gas drag presented by the solar nebula, kmsized planetesimals formed in the giant planet zone are unlikely to be gravitationally scattered outward in order to populate the well-known cometary reservoirs (Brasser et al. 2007). Thus, the trans-planetary space, hosting a massive disk of planetesimals, is currently favored as the birth place of comet nuclei.

The issue of collisional evolution among those planetesimals has been discussed for almost 20 yr. Davis \& Farinella (1997) modeled the collisional evolution of the Edgeworth-Kuiper belt (EKB) and argued that its members with radii of a few $\mathrm{km}$ - like typical Jupiter family comets - are multigenerational fragments formed by the splitting of larger objects. Stern \& Weissman (2001) considered the formation of the Oort cloud out of planetesimals formed in the giant planet zone. They showed that, during the course of this gravitational scattering process, comet nuclei would be destroyed by collisions with small debris. However, following the introduction of the Nice model for the evolution of the solar system (Tsiganis et al. 2005), the EKB is no longer thought to be the direct survivor of the initial comet population $^{1}$, and according to Brasser \& Morbidelli (2013), the actual Oort cloud formation may have little to do with the classical concept of scattering of planetesimals from the formation zone of the giant planets.

In this paper we discuss some of the evidence that OSIRIS imaging of the 67P nucleus may bring regarding its origin and evolution. We argue that the bi-lobate shape is likely due to a gentle merger of the two lobes as separate bodies in a lowvelocity collision. This conclusion is supported by additional observations, such as the presence of thick layering and fracturing. We place this into the framework of the collisional evolution issue as it currently stands, and we highlight the importance of resolving the remaining issues about the fundamental question, whether the 67P nucleus is the result of an early merging of two distinct planetesimals or was re-accreted in connection

\footnotetext{
1 The cold EKB may indeed be a remnant of the primordial disk, but it carries a very small fraction of the total mass (Fraser et al. 2014).
}

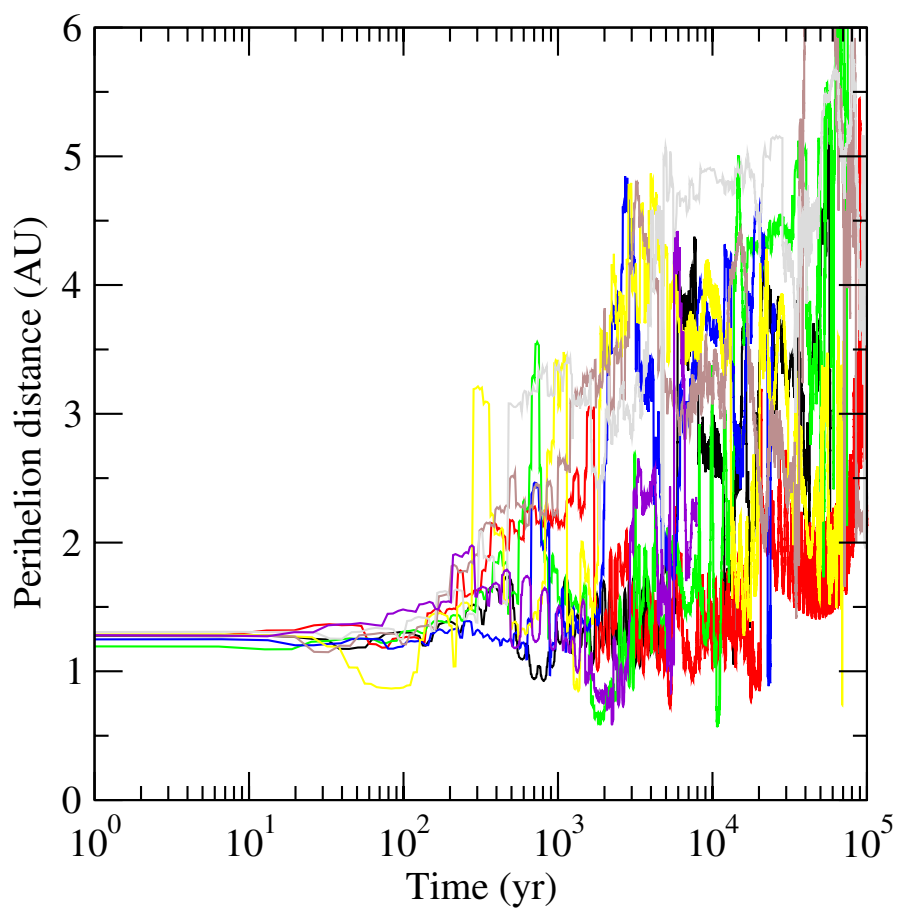

Fig. 1. Evolutions of the perihelion distance of 67P, found by forward integrations of random initial orbits leading into current orbits close to the one of 67P. The time plotted on the abscissa is counted backward from the present. The different colors mark the different examples chosen. We note that these are not clones of $67 \mathrm{P}$, and the recent history of 67P (including the close encounter with Jupiter in 1959) is not reproduced. The focus is on the long-term dynamics, shown in the right part of the diagram. Chaotic divergence of the backward motions sets in, mostly a few centuries ago, due to close encounters with Jupiter. Courtesy Ryszard Gabryszewski.

with a major collision long after the planetesimals were formed. Meanwhile, we also point out that, in any case, this nucleus does not appear to have completely lost its primordial signatures and may thus retain its significance as a witness of planetesimal formation in the nascent solar system.

In Sect. 2 we discuss the role of past erosion in shaping the $67 \mathrm{P}$ nucleus and evidence regarding its bi-lobate shape and the interpretation as a contact binary. We summarize the evidence concerning structural features of the nucleus and the origin of the contact binary in a low-velocity collision. Section 3 gives an estimate of the collision rate in the trans-planetary disk, which illustrates that it is generally unlikely for km-sized planetesimals to survive intact during the expected lifetime of this disk, and in Sect. 4 we discuss possible ways for comets to escape destruction in the event that $67 \mathrm{P}$ should prove to be such a survivor. Our conclusions are summarized in Sect. 5.

\section{Overall shape and structural features of $67 \mathrm{P}$}

\subsection{Previous erosion}

Thermo-physical models reveal that 67P could have lost a surface layer of up to several hundred meters thickness due to the accumulated activity (Sierks et al. 2015; Keller et al. 2015) during its previous orbits in the inner solar system. This estimate is based on the median number of such orbits as estimated from long-term integrations for fictitious objects similar to 67P, and the specific evolutions of the perihelion distance of the comet, which largely governs the erosion rate. Figure 1 shows an 
example of the large variety of previous evolutions of the perihelion distance of 67P that may lead into orbits close to the present one according to long-term integrations. In general, these evolutions have brought the comet's perihelion closer to the Sun, but Fig. 1 covers only part of the whole time spent by the comet in the inner solar system. In many cases, the comet had made earlier visits into orbits with small perihelion distance, which also contributed to the cumulative erosion. We note that the dynamical time scale found by these integrations may be consistent with the capture time scale recently estimated by Guzzo \& Lega (2015), although further work is needed to confirm this.

While the use of the current shape and spin axis orientation cannot have a drastic influence on those results, the extrapolation of the current activity level may possibly be an important source of error. In particular, there is a concern about the possibility that the comet has undergone long periods of dormancy (Kresák 1987), when its gas production was close to zero. This has been suggested for individual comets - for instance, Levison et al. (2006) found that the orbital properties of 2P/Encke may be explained, if this comet spent a long time in the past as a dormant comet and was relatively recently woken up, as its perihelion distance was decreased by a secular resonance.

In case dormancy can become permanent, i.e., comets stay dormant until they are dynamically ejected, this may influence our estimate of past erosion for the reason that the current activity of $67 \mathrm{P}$ would indicate the comet to be relatively young in the dynamical sense. This youth is of course not verified by long-term orbital integrations due to the strongly chaotic behavior, i.e., short Lyapunov time scales, of Jupiter family comets. Permanent dormancy would seem to be supported by the work of Duncan \& Levison (1997), who concluded that the physical lifetime of these comets must be limited because their orbits retain a memory of the very low inclinations in the scattered disk that they assumed as the source for captures. However, the scattered disk is now recognized to be much more excited, so this argument may not be of relevance any more.

We tentatively conclude that a thick surface layer has likely been eroded from the 67P nucleus by previous gas-producing activity, although the above-mentioned estimate of several hundred meters should perhaps be regarded as an upper limit. In any case, this cautions that activity-driven erosion may result in complex landscapes (Malin \& Zimbelman 1986; Thomas et al. 2005), which could affect our ability to interpret geomorphological features in terms of primordial processes. This is particularly true for equatorial regions and the southern hemisphere due to their computed higher erosion rates (Keller et al. 2015). Furthermore, it should be noted that the small-scale details seen on the surface of the 67P nucleus are likely of very recent origin in most cases. For instance, because 67P currently crosses the main asteroid belt, following the methodology developed by Marchi et al. (2010, 2012a,b) we estimate that some 100-1000 craters larger than a meter should have formed over a time scale of $10^{3} \mathrm{yr}-$ however, these craters are not observed.

For these reasons, we will focus our attention on the overall bi-lobate shape and the large-scale structural properties of two key regions, Seth and Hathor (Thomas et al. 2015), which are least affected by activity-driven erosion (Keller et al. 2015). These regions face the "neck" from the larger and smaller lobes, respectively.

\subsection{Contact binary}

The bi-lobate shape of 67P has important implications for the formation of the nucleus. Computations based on the current shape, spin axis orientation, and a homogeneous composition show that the neck-facing regions (in particular, the Seth and Hathor regions) are not subject to an increased dose of solar radiation that would imply preferential erosion (Sierks et al. 2015; Keller et al. 2015). Although it cannot be ruled out that 67P had a radically different spin axis orientation in the past, it seems unlikely that erosion of a homogeneous object alone could explain the current shape of 67P. It is nevertheless possible that a very heterogeneous nucleus (with locally higher concentration of volatiles, or different terrain properties) could result in preferential, local erosion. Such a heterogeneous structure, however, may require mixing of planetesimals with different volatile compositions or abundances, probably only attainable through collision and merging of objects formed at different heliocentric distances.

Alternatively, the bi-lobate shape may strongly suggest 67P to be a contact binary. While contact binaries have been advocated for elongated comet nuclei (e.g., 1P/Halley, 19P/Borrelly and $103 \mathrm{P} /$ Hartley 2 ), the only unquestionable case remains that of $8 \mathrm{P} /$ Tuttle based on radar imaging (Harmon et al. 2010) and indirectly confirmed by HST observations (Lamy et al. 2008). Indeed, based on its appearance, 67P is a highly likely candidate. As such, however, its shape appears unusual, since the two lobes have their longest axes nearly perpendicular whereas they are aligned in most if not all other contact binaries.

Binary systems are ubiquitous in various small-body populations, including the near-Earth asteroids (NEAs), main-belt asteroids, Jupiter Trojans, Centaurs, and trans-Neptunian objects (TNOs). Contact binaries alone are estimated to constitute 10-20\% of all NEAs, Trojans, and TNOs (Sheppard \& Jewitt 2004; Noll et al. 2008; Mann et al. 2007; Benner et al. 2006, 2008). Several mechanisms for the formation of binary systems have been proposed, ranging from fission of small rapidly rotating NEAs (Scheeres 2007), to the formation of large binary TNOs by gravitational collapse (Nesvorný et al. 2010). Whether or not these processes are applicable to highly porous, small comets deserves further investigation.

\subsection{Structural features}

Concerning possible structural features, the Seth region displays multiple semi-planar terrains, or facets (Thomas et al. 2015; Massironi et al. 2015). Planar terrains are, in some cases, reminiscent of what has been seen on other comet nuclei (e.g., Belton et al. 2007). A key difference, however, is that Seth is characterized by the presence of deep collapsed pits (Vincent et al. 2015), whose dust-free walls allow us to glance at the local vertical stratigraphy. In many instances, these walls reveal quasiparallel lineaments and small terraces, which may be interpreted as the surface expression of internal layering (Marchi et al. 2015; Massironi et al. 2015).

In the case of the largest terrace in the Seth region, a shape model (Jorda et al., priv. comm.) was used to fit the floor of the pit with a plane to obtain an approximate local orientation of the putative layer (Figs. 2a, b). Interestingly, the orientation of the resulting plane, extrapolated to the extreme end of the large lobe, matches the orientation of a topographic ridge used to mark the boundary of two very morphologically distinct terrains (Ash and Imhotep regions; Thomas et al. 2015; Figs. 2c,d), suggesting that the flat floors and lineaments are truly indicative of internal layering, with an estimated local depth of at least a few hundred meters (see Marchi et al. 2015; Massironi et al. 2015).

The Seth region faces a prominent dust-free cliff in the small lobe, named Hathor, which reveals the ubiquitous presence of 

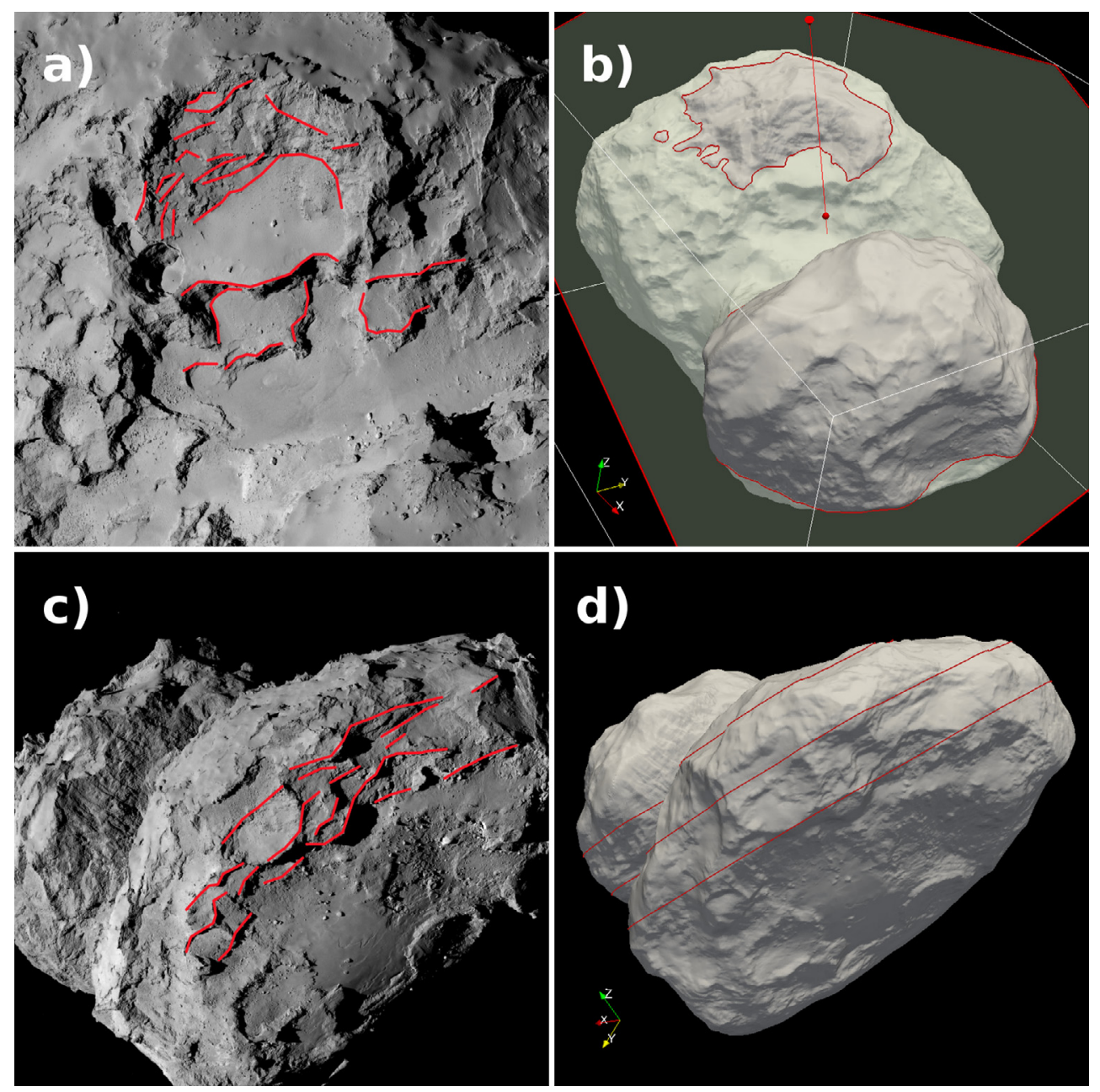

Fig. 2. Large-scale layering on the large lobe. a) Close-in view of the pitted terrain on the large lobe (Seth region). The red lines indicate some of the prominent lineaments, interpreted as surface expression of layering. b) A plane indicating the prevailing local orientation of the layers for the largest pit in the Seth region. c) A set of lineaments and high topographic reliefs on the opposite side of the large lobe. d) Same plane as in b) and two additional parallel planes. The orientation of these planes matches the lineaments and topographic reliefs, suggesting the latter are surface expressions of internal layering. We note that the local orientation of the plane may vary from place to place. For simplicity we assumed that the best fit plane does not change attitude within the large lobe. However, a similar conclusion applies for bending following the local convex shape, or along the $y$-axis in panel $\mathbf{b}$ ).

fractures and terraces, the latter being interpreted as the result of layering (Fig. 3a). The two sets of layering in the Seth and Hathor regions have local spatial orientations that are not compatible with being the expression of a homogeneous layering wrapping the two lobes (Marchi et al. 2015; Massironi et al. 2015). A similar conclusion is also reached by looking at the global distribution of semi-planar terrains on the surface imaged so far (Massironi et al. 2015).

The Hathor region also hosts another large-scale geological feature with possible implications for the formation of 67P, i.e., an impressive set of fractures that run for hundreds of meters from the base of the small lobe to its summit (Figs. 3a,b). The largest of these are inferred to be several meters wide, based on the cast shadows. Several hypotheses for their formation have been investigated, such as thermal fracturing, and gradual sandblasting erosion due to activity from the neck region. Thermal fracturing typically results in polygonal patterns, such as those observed in permafrost on Mars and Earth (e.g., Levy et al. 2010), and is therefore an unlikely explanation for this wellorganized set of large fractures. Sand-blasting from a cometary jet generally exerts a pressure much lower than the relevant material strength, and therefore also does not appear to be a likely mechanism.

Alternatively, wide spread fracturing could be achieved through large-scale torques due to re-orientation of the two lobes, or by rotational stresses exceeding the failure strength. However, such torque-induced fractures are expected to form in the neck region itself, where most of the resulting stresses would concentrate, rather than the neck-facing regions. Moreover, given the almost symmetric nature of the Seth and Hathor regions with respect to the neck, such processes should result in similar fracturing. While some degree of fracturing is indeed observed in the Seth region, the lack of an extensive and wellorganized system of fractures suggests that the two lobes may have distinct properties.

In addition, a spatial analysis of the orientations of the fractures suggests they penetrate deeply into the small lobe, indicating that they may be pervasive. Support to this conclusion comes from the morphology of a large depression, $\sim 1 \mathrm{~km}$ across, at the summit of the small lobe, named Hatmehit region (Thomas et al. 2015). Whether this depression is the result of a collapse or an outburst, its rhombic shape indicates the presence of planes of 

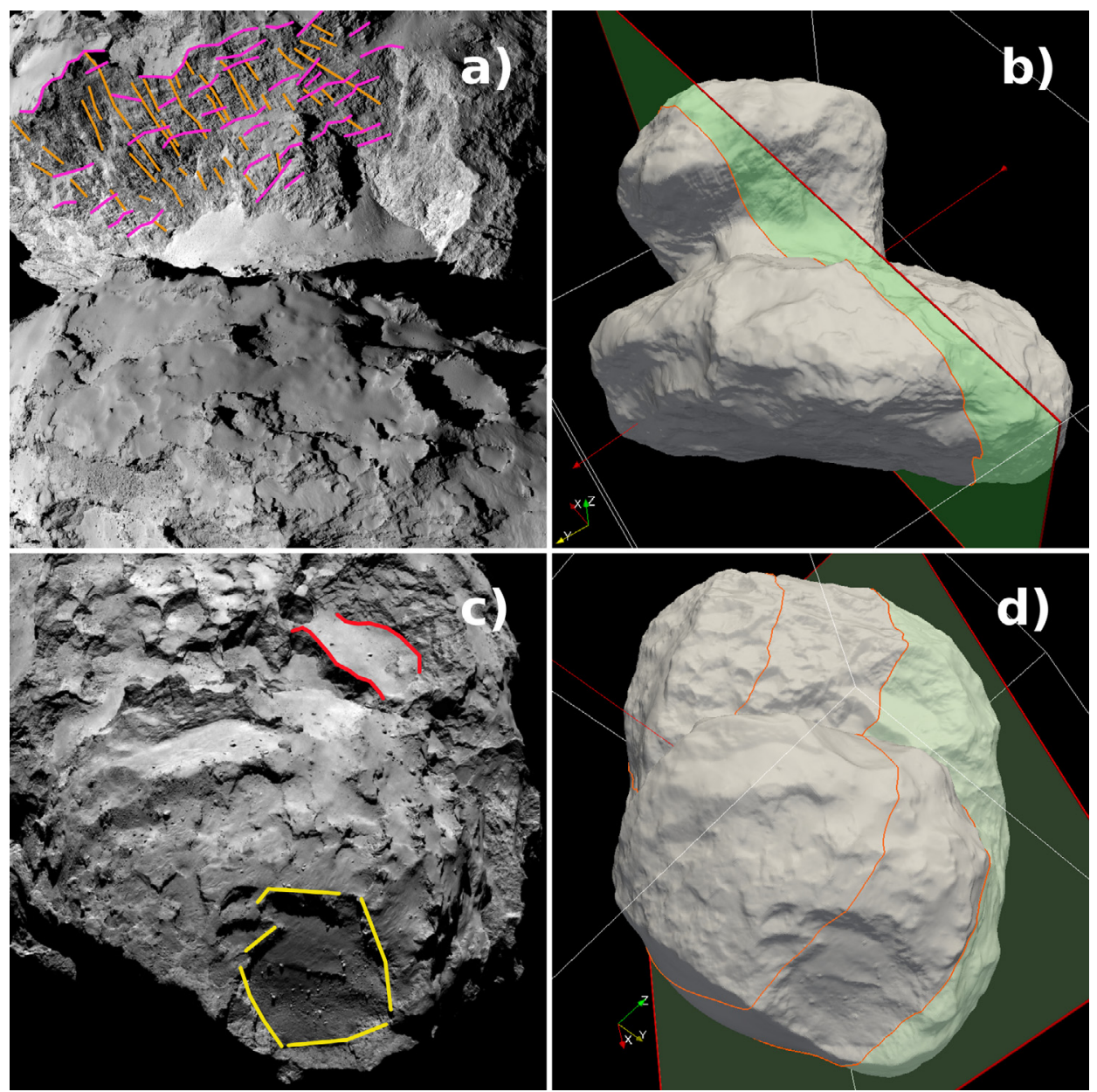

Fig. 3. Large-scale fractures on the small lobe. a) Cross-cutting pattern of fractures (orange lines) and layers (purple lines) on the Hathor region. b) A plane indicating the prevailing orientation of the fractures overlaid on a shape model. c) A view of the top of the small lobe, with some major features highlighted: layering on the large lobe (red lines; see Fig. 2), rim of the Hatmehit depression (yellow lines). d) The same plane shown in b), and an additional parallel plane. The orientation of these planes matches the direction of two sides of the Hatmehit depression.

weakness within the small lobe, which appear to be aligned with the Hathor fracture system (Figs. 3c, d). It therefore seems possible that these fractures are structural features, pervading the smaller lobe.

\subsection{Binary formation}

The above geomorphological evidence on 67P supports the view that the bi-lobate nature of $67 \mathrm{P}$ is due to collision and merging of two distinct objects. Further constraints on this event can be derived from the high porosity of 67P (Sierks et al. 2015) and the content of volatile species. The high porosity requires peak pressures likely not exceeding $\sim 100 \mathrm{kPa}$ (Yasui \& Arakawa 2009), and hence an accretional speed not exceeding a few tens of $\mathrm{m} / \mathrm{s}$. The average impact speed of small planetesimals is thought to have evolved from $\sim 10 \mathrm{~m} / \mathrm{s}$ during the early stages still dominated by gas (Kokubo \& Ida 2000) to $\sim 500 \mathrm{~m} / \mathrm{s}$ corresponding to viscous stirring of the planetesimal disk by its largest members (Levison et al. 2011). These considerations narrow down two possible formation scenarios for $67 \mathrm{P}$.

In one case, $67 \mathrm{P}$ is the result of a low-speed collision between two planetesimals, which most likely took place in the earliest stages of the disk evolution before gas dispersal. This would mean that the structure of $67 \mathrm{P}$ dates back to the time of planetesimal formation and has not been significantly altered by later evolution. Alternatively, 67P is the result of re-accumulation of fragments ejected during a catastrophic collision involving a larger planetesimal. Numerical simulations (e.g., Jutzi et al. 2010) have shown that the catastrophic disruption of porous parent bodies may result in ejection velocities $\$ 10 \mathrm{~m} / \mathrm{s}$ (comparable to the mutual impact speed among fragments), while for a typical impact speed of $\sim 500 \mathrm{~m} / \mathrm{s}$ or lower a significant volume of the parent body may experience low shock pressures (e.g., Leinhardt \& Stewart 2009, rescaled to lower impact speeds). In addition, a highly porous parent body will greatly help damping the shock wave, therefore reducing the pressure exerted far from the impact location (Wünnemann et al. 2006). Therefore, the collisional scenario should also be kept in consideration as a possible mode of origin of 67P. In this case too, the structure formed during the re-accumulation must not have been destroyed by subsequent evolution, but preceding collisions on the same parent body cannot be excluded.

Our discussion suggests that the two lobes of 67P are two distinct objects that merged in a low-speed collision. However, the issue remains, whether this collision was part of the primordial planetesimal accretion, or if it involved fragments produced later by a collision experienced by a larger parent body. This is very important, not only to realize which scenario would best explain the properties of 67P as observed by OSIRIS and other instruments, but also because current concepts on solar system 
evolution have important implications for the collisional evolution of comets. We shall now turn to the latter.

\section{Collisional evolution in the trans-planetary disk}

\subsection{General framework}

We place our discussion within the current paradigm of solar system evolution as defined by the Nice model (Tsiganis et al. 2005; for the latest development see Levison et al. 2011). This model features a disk of planetesimals beyond the initial, planetary orbits, which should have extended roughly from 15 to $30 \mathrm{AU}$ in heliocentric distance. The disk was formed in the nascent solar system, when the planetesimals were formed, and it was dispersed as a consequence of planet migration following the dynamical instability of the giant planets.

The dispersal is thought to have taken place about 4.1-4.2 Gy ago (Morbidelli et al. 2012; Marchi et al. 2013), implying $\sim 0.4$ Gy of prior collisional evolution. The total mass of the disk is constrained to be in the range 20-50 Earth masses (Tsiganis et al. 2005). The timing of the dynamical instability is assumed to coincide with the start of the late heavy bombardment (LHB). Among the important effects of the instability, there is also the gravitational scattering of objects into the Oort cloud and the scattered disk (Brasser \& Morbidelli 2013). We consider that, at the typical velocities of collisions in the trans-planetary disk, projectiles with diameter $(D)$ larger than $1 \mathrm{~km}$ would shatter targets of the same size as $67 \mathrm{P}(D \sim 4 \mathrm{~km}$, assuming a past erosion of a $300 \mathrm{~m}$ thick layer). This estimate is likely conservative, judging from the expected material properties (e.g., Benz \& Asphaug 1999) ${ }^{2}$.

Concerning the availability of such projectiles, a recent study by Johansen et al. (2015) of planetesimal formation in a pebble cloud found the cumulative size frequency distribution (SFD) to be quite shallow with a power law index $\alpha \simeq-1.8$ for $20 \lesssim D \lesssim 200 \mathrm{~km}$, although the applicability of this shallow slope down to km-sized objects needs to be further investigated. Additional constraints on this size range come from the observed trans-Neptunian populations and Trojan asteroids (the current leftovers of the primordial disk). These populations exhibit twosloped cumulative SFDs, with a break at $D \sim 100 \mathrm{~km}$. The slope of the cumulative SFDs for smaller objects ranges from -2.0 to -2.5 (Grav et al. 2011; Fraser et al. 2014). Of course, if the planetesimal population evolves by collisions, new small objects are likely produced as fragments of larger ones. This is guaranteed by the above-mentioned shallow slopes of the planetesimal SFD, while it would not hold true for a much steeper planetesimal SFD of the kind suggested by Belton (2015).

\subsection{Number of collisions in the trans-planetary disk}

The number of disruptive collisions $\left(N_{\text {coll }}\right)$ for $67 \mathrm{P}$ can be estimated using a particle-in-a-box formula (Wetherill 1967),

$$
N_{\text {coll }}=\frac{N_{\mathrm{p}} u T A_{\mathrm{p}}}{V},
$$

\footnotetext{
2 Morbidelli \& Rickman (2015) used the Benz \& Asphaug (1999) scaling law for hard ice, hit at $1 \mathrm{~km} \mathrm{~s}^{-1}$, and found that the minimum diameter of a disruptive projectile for a target of the same size as 67P was always about $1 \mathrm{~km}$ or less throughout the primordial disk. However, for porous targets the minimum projectile diameter would be smaller, using the weak ice scaling law of Leinhardt \& Stewart (2009).
}

where $N_{\mathrm{p}}$ is the number of disk objects, $A_{\mathrm{p}}=\pi\left(R_{\mathrm{c}}+R_{\mathrm{p}}\right)^{2}$ is the cross section for a collision between $67 \mathrm{P}$ with average radius $R_{\mathrm{c}}=2 \mathrm{~km}$ and a partner with radius $R_{\mathrm{p}}, u$ is the average relative velocity, $T$ is the time interval, and $V$ is the volume of the disk. Assuming viscous stirring of the disk by its largest members (Levison et al. 2011), we estimate $u=0.6 \mathrm{~km} \mathrm{~s}^{-1}$, which is $10 \%$ of the typical orbital velocity in the disk. The volume is obtained by considering a circular annulus with inner and outer radii of 15 and $30 \mathrm{AU}$, and a constant thickness of $4 \mathrm{AU}$.

As collision partners we consider disk objects with radius from $0.5 \mathrm{~km}$ up to the break point in the cumulative planetesimal SFD, which occurs at $R_{\mathrm{p}} \sim 50 \mathrm{~km}$. We take a power law with constant index $\alpha$ for the cumulative planetesimal SFD and derive the corresponding differential number $n\left(R_{\mathrm{p}}\right) \mathrm{d} R_{\mathrm{p}}$ of disk objects in a radius range of width $\mathrm{d} R_{\mathrm{p}}$. We replace $N_{\mathrm{p}} A_{\mathrm{p}}$ in Eq. (1) by the integral

$$
\int n\left(R_{\mathrm{p}}\right) A_{\mathrm{p}} \mathrm{d} R_{\mathrm{p}}
$$

taking the limits at $0.5 \mathrm{~km}$ and $50 \mathrm{~km}$. The computation of $n\left(R_{\mathrm{p}}\right)$ will first be done as follows. At the end of the time interval $(T=0.4 \mathrm{~Gy})$ we use an estimate of $2 \times 10^{11}$ objects with $D>2.3 \mathrm{~km}$ in order to populate the scattered disk (Brasser \& Morbidelli 2013). Populating the Oort cloud would seem to require even more, but we prefer to be conservative. For the cumulative SFD index $\alpha$ we consider two values: -2.5 , which would correspond to a relaxed, collisional asteroid population (Dohnanyi 1969), and -2.0, which is close to the value found by a theoretical model (Johansen et al. 2015) for small planetesimals formed by pebble accretion. The latter would likely characterize the initial state of the disk, and the former may characterize the final state after collisional evolution.

For the sake of illustration, we use the above value for the cumulative number with $D>2.3 \mathrm{~km}$ with the two $\alpha$ values to characterize the disk throughout the time interval, even though it explicitly refers to the final state. Our calculation then yields $N_{\text {coll }} \simeq 11$ and 9 for $\alpha=-2.5$ and -2.0 , respectively. In that case, 67P-sized objects would undergo about ten collisions on the average, and the chance to survive without any collision, as estimated by a Poisson distribution, is less than $10^{-4}$. Thus, for 67P to survive undisrupted, the initial $N_{\mathrm{p}}$ would have to be at least a factor 10 smaller than we have assumed. Hence, the final objects would have to be mostly collisional fragments, and it is statistically unlikely for 67P to be among the initial survivors.

Interestingly, the disk mass in the considered size range, assuming a density of $1000 \mathrm{~kg} / \mathrm{m}^{3}$ (Brown 2013), comes out as 6 or 18.5 Earth masses $\left(M_{\mathrm{E}}\right)$ for the two $\alpha$ values. We now consider the contribution by objects with $D>100 \mathrm{~km}$. The slope of the cumulative SFDs for the trans-Neptunian populations and Trojan asteroids for diameters larger than $\sim 100 \mathrm{~km}$ ranges from -3.5 to -4.5 . We will use $\alpha=-4$ for the larger objects (Gladman et al. 2001; Fraser et al. 2014) while keeping both limits discussed above for the smaller size range as in the previous computation. Very large objects (such as Pluto) are insufficiently sampled by the adopted planetesimal SFD, but it is expected that $\sim 1000$ Pluto-sized objects once existed in the primordial disk (Levison et al. 2011), thus contributing a few Earth masses. With these assumptions, the total mass is compatible with the inferred range from the Nice model as stated above.

An alternative way of estimating $n\left(R_{\mathrm{p}}\right)$ is as follows. We take the minimum value of $20 M_{\mathrm{E}}$ for the total disk mass. We subtract $2 M_{\mathrm{E}}$ for the Pluto-sized objects and consider $18 M_{\mathrm{E}}$ to be partitioned between two populations with $D<100 \mathrm{~km}$ and $D>100 \mathrm{~km}$. We distribute the $18 M_{\mathrm{E}}$ up to $D=1000 \mathrm{~km}$, and 


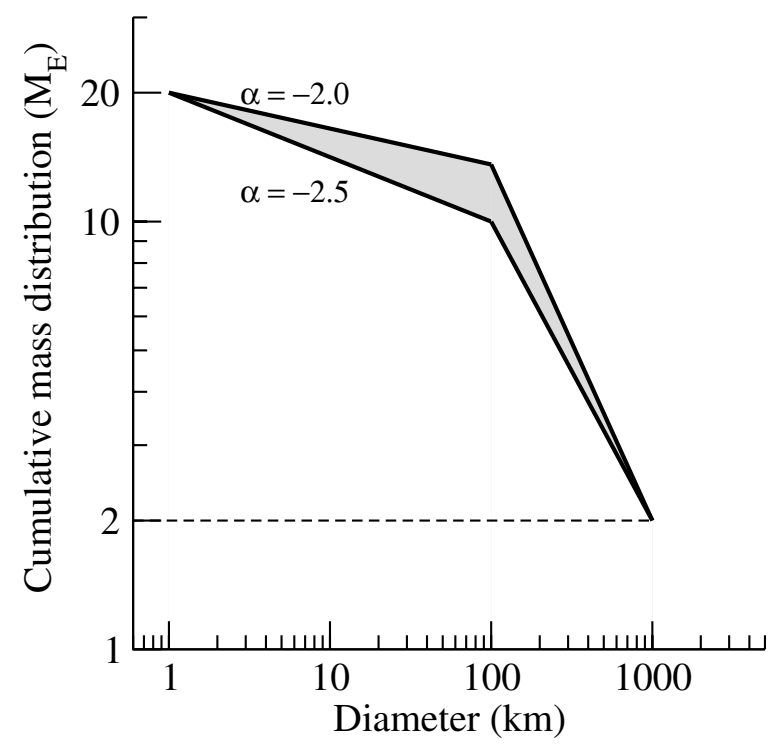

Fig. 4. A sketch of the primordial disk cumulative mass distribution (in units of Earth's masses), based on dynamical constraints and current observational constraints from Kuiper belt objects and Trojan asteroids. We plot, as a function of diameter, the total disk mass contained in all larger objects. Two Earth masses have been allocated to objects larger than $1000 \mathrm{~km}$. The total mass of objects larger than $1 \mathrm{~km}$ has been set to 20 Earth masses, at the low end of published primordial disk masses. The two solid curves correspond to slopes of -2.0 and -2.5 for the cumulative size-frequency distribution at $D<100 \mathrm{~km}$.

as a result we find for $\alpha=-2.5$ that the number of objects with $D>2.3 \mathrm{~km}$ is $3 \times 10^{11}$, yielding $N_{\text {coll }} \simeq 18$, and for $\alpha=-2.0$ we find $7 \times 10^{10}$, yielding $N_{\text {coll }} \simeq 3$. In this case, even for the shallower cumulative planetesimal SFD, the chance for survival without any collision is only 5\%, and yet the planetesimal population has an insufficient number of objects to feed the scattered disk. Figure 4 illustrates the mass distribution used for the transplanetary disk objects in this analysis.

\section{Escape from collisions}

We conclude from the above that in both scenarios under consideration, 67P is likely to be collisionally evolved. To judge the chances for 67P to be unaffected and primordial, we may consider a possible gradient of collision probability across the disk. Its surface density is usually modeled to vary as $r^{-1}$ with heliocentric distance $r$. In addition, the thickness of the disk may have been proportional to $r$, so that the number density may have varied as $r^{-2}$. This would cause $N_{\text {coll }}$ to be four times lower in the outermost parts of the disk than in the innermost parts. Therefore, 67P may possibly be a primordial survivor without being too exceptional, if it formed in the outskirts of the disk, as possibly indicated by its high $\mathrm{D} / \mathrm{H}$ ratio (Altwegg et al. 2015). In addition, an even lower mass of the disk (Nesvorný, priv. comm.), and a less dynamically excited disk could also reduce the estimated collision rate by a few times. In any case, it appears that the best chance for $67 \mathrm{P}$ to be primordial is with a low-mass disk and a shallow size distribution of the planetesimals (namely, $\alpha \lesssim-2$ for $D<100 \mathrm{~km}$ ).

Our model features a common value of the encounter velocity for the trans-planetary disk objects. This is thought to be an average of the individual velocities, relevant to the average state of dynamical excitation of the disk. Of course, there are many encounters occurring at both lower and higher velocities, so we should consider the effect of this scatter. Since we used a cut-off of projectile diameters at $D=1 \mathrm{~km}$, we cannot account for the fact that the size limit for disruptive collisions decreases with increasing velocity. However, this together with the fact that the collision frequency increases with velocity means that we have likely underestimated the number of disruptive collisions.

Another caveat concerns the timing of the giant planet instability. Even though it is considered likely that the LHB was triggered by the flux of asteroids and comets destabilized by the planet migration, there remains a possibility that the instability occurred earlier than assumed here. In such a case, the disk lifetime may have been significantly less than $400 \mathrm{My}$, thus drastically lowering the above estimates of $N_{\text {coll }}$. However, a very early instability would face other problems such as finding an alternate explanation for the LHB.

We should also keep in mind that, whatever was the fate of the planetesimals from which 67P stems, our estimates confirm the recent conclusion by Morbidelli \& Rickman (2015) that comets in general did not escape collisional evolution in the early solar system. This holds for all kinds of comets - those coming from the Oort cloud as well as those captured from the scattered disk or the EKB. Therefore, if 67P and other comets are found to retain primordial structural features, it follows that these cannot have been erased by the collisional history of the comets.

In this perspective, 67P appears to offer a precious test case, since it has not stood out as very special among the JFCs. Thus, the study of a collisional re-accretion origin for $67 \mathrm{P}$ is particularly important. For instance, should such an origin prove to be inconsistent with the body of evidence from OSIRIS and other Rosetta instruments in the sense that the primordial signatures (e.g., layering and outgassing of super-volatiles) would have been erased, this would suggest that both $67 \mathrm{P}$ and comets at large, are indeed primordial survivors. Such a conclusion would necessitate a reconsideration of the concepts around the early evolution of the solar system that were applied in this paper.

On the other hand, should it turn out that collisional reaccretion is consistent with the observations of 67P including those features thought to pre-date this accretion (e.g., layering and volatile abundances), the study of $67 \mathrm{P}$ would still reveal important clues about early planetesimal formation, independent of the role played by collisions in its later history.

\section{Discussion and conclusions}

We have shown that OSIRIS data helps to constrain the origin of comet 67P/Churyumov-Gerasimenko in several important ways. The bi-lobate overall shape is best interpreted in terms of a contact binary, formed by the collisional merger of two distinct bodies. Their properties appear generally similar, but the smaller lobe may possibly show evidence of damage due to an energetic impact, perhaps witnessed by the observed large-scale fractures.

The significant porosity inferred from a revised bulk density of $\sim 510 \mathrm{~kg} / \mathrm{m}^{3}$ (L. Jorda, 2015, priv. comm.) in fact requires accretional velocities for the two lobes $\lesssim 10-20 \mathrm{~m} / \mathrm{s}$, and this leaves open two options for the origin of 67P. Either it may be an undestroyed, primordial planetesimal that accreted during the first $\sim 10 \mathrm{My}$, or it was formed by the gentle re-accretion of fragments ejected from a larger parent body upon a destructive collision at a later time (possibly, the last one of a series of collisions). It is not possible yet to exclude either of these scenarios. However, should the latter option be true, it must have conserved the primordial signatures of 67P (both geomorphological and chemical), and hence the comet remains a key witness of planetesimal formation in the nascent solar system. We also emphasize that 
the collisional scenario requires the bi-lobate nucleus to have survived intact after it was formed, and thus the collision that led to its formation must have occurred during the final stages of the primordial disk or even during its dispersal.

The origin of internal layers remains elusive. However, it should be noted that layering appears to be an inherent characteristic of the two lobes prior to their accretion. According to Groussin et al. (2015), the low compressional strength of 67P may stimulate pressure-induced layering. The early, gentle docking of two km-sized planetesimals thus implies that their layering arose during their primordial accretion. Alternatively, the involvement of a catastrophic disruption of a larger parent body (a few $10 \mathrm{~s} \mathrm{~km}$ at most to avoid significant reduction of porosity by compaction) could explain layering, if some internal evolution had taken place resulting in an onion-shell structure, and the two lobes are large chunks of the parent body rather than debris piles.

We also show that strong collisional evolution is generally to be expected for the objects that occupied the early, transplanetary disk. While it is possible for 67P to have survived collisional destruction in a safe niche under special assumptions about the disk, we point out that 67P has not shown any evidence of being exceptional among comets at large, and thus it should not have an exceptional origin. However, this also means a challenge for the collisional origin scenario. Low densities have been found for all Jupiter family comets so far analyzed, and if these are generally produced by destructive collisions involving large parent bodies, the latter must have conserved deep surface layers in a primordial, porous state in spite of ${ }^{26} \mathrm{Al}$-induced chemical differentiation (Prialnik et al. 2004). Interestingly, several EKB objects in the size range $D \sim 150-400 \mathrm{~km}$ have been found to have a density of $400-800 \mathrm{~kg} / \mathrm{m}^{3}$ (Brown 2013), implying significant porosity can be retained even on large objects.

Regarding the two main options, we conclude that the primordial survivor option would likely call into question some of the current concepts of solar system evolution, while the collisional fragment option would not call for any such rethinking. The issue with the latter is to show it to be consistent with the observational evidence on 67P and other comets.

Acknowledgements. The authors wish to thank Hal Levison, Alessandro Morbidelli, Katherine Kretke, David Nesvorný, Alex Parker, Robert Grimm, Anders Johansen for interesting discussions. We also thank the referee, David O'Brien, for helpful suggestions. OSIRIS was built by a consortium led by the Max-Planck-Institut für Sonnensystemforschung, Göttingen, Germany, in collaboration with CISAS, University of Padova, Italy, the Laboratoire d'Astrophysique de Marseille, France, the Instituto de Astrofísica de Andalucia, CSIC, Granada, Spain, the Scientific Support Office of the European Space Agency, Noordwijk, Netherlands, the Instituto Nacional de Técnica Aeroespacial, Madrid, Spain, the Universidad Politéchnica de Madrid, Spain, the Department of Physics and Astronomy of Uppsala University, Sweden, and the Institut für Datentechnik und Kommunikationsnetze der Technischen Universität Braunschweig, Germany. The support of the national funding agencies of Germany (DLR), France (CNES), Italy (ASI), Spain (MEC), Sweden (SNSB), and the ESA Technical Directorate is gratefully acknowledged. We thank the ESA teams at European Space Astronomy Center, European Space Operations Center, and European Space Research and Technology Center for their work in support of the Rosetta mission. Special thanks are due to Ryszard Gabryszewski (PAS Space Research Center, Warsaw) for permission to use the plot in Fig. 1. S.M. was supported by NASA Jet Propulsion Laboratory Subcontract No. 1336850 to the Southwest Research Institute. H.R. was supported by Grant No. 2011/01/B/ST9/05442 of the Polish National Science Center.

\section{References}

Altwegg, K., Balsiger, H., \& Bar-Nun, A. 2015, Science, 347, 387 Belton, M. J. S. 2015, Icarus, 245, 87

Belton, M. J. S., Thomas, P., Veverka, J., et al. 2007, Icarus, 187, 332

Benner, L.-A. M., Nolan, M. C., Ostro, S. J., et al. 2006, Icarus, 182, 474

Benner, L.-A. M., Nolan, M. C., Margot, J.-L., et al. 2008, BAAS, 40, 432

Benz, W., \& Asphaug, E. 1999, Icarus, 142, 5

Brasser, R., \& Morbidelli, A. 2013, Icarus, 225, 40

Brasser, R., Duncan, M. J., \& Levison, H. F. 2007, Icarus, 191, 413

Brown, M. E. 2013, ApJ, 778, L34

Davis, D. R., \& Farinella, P. 1997, Icarus, 125, 50

Dohnanyi, J. S. 1969, J. Geophys. Res., 74, 2531

Duncan, M. J., \& Levison, H. F. 1997, Science, 276, 1670

Fraser, W. C., Brown, M. E., Morbidelli, A., Parker, A., \& Batygin, K. 2014, ApJ, 782, 100

Gladman, B., Kavelaars, J. J., Petit, J.-M., et al. 2001, AJ, 122, 1051

Grav, T., Mainzer, A. K., Bauer, J., et al. 2011, ApJ, 742, 40

Groussin, O., Jorda, L., Auger A.-T., et al. 2015, A\&A, 583, A32

Guzzo, M., \& Lega, E. 2015, ArXiv e-prints [arXiv: 1307.6731]

Harmon, J. K., Nolan, M. C., Giorgini, J. D., \& Howell, E. S. 2010, Icarus, 207, 499

Johansen, A., Blum, J., Tanaka, H., et al. 2014, in Protostars and Planets VI, eds. H. Beuther, R. S. Klessen, C. P. Dullemond, \& T. Henning (Tucson: Univ. Arizona), 547

Johansen, A., Mac Low, M. M., Lacerda, P., \& Bizzarro, M. 2015, Sci. Adv., 1, 1500109

Jutzi, M., Michel, P., Benz, W., \& Richardson, D. C. 2010, Icarus, 207, 54

Keller, H. U., Mottola, S., Davidsson, B., et al. 2015, A\&A, 583, A34

Kokubo, E., \& Ida, S. 2000, Icarus, 143, 15

Kresák, L. 1987, A\&A, 187, 906

Lamy, P. L., Toth, I., Jorda, L., et al. 2008, BAAS, 40, 393

Leinhardt, Z. M., \& Stewart, S. T. 2009, Icarus, 199, 542

Levison, H. F., Terrell, D., Wiegert, P. A., Dones, L., \& Duncan, M. J. 2006, Icarus, 182, 161

Levison, H. F., Morbidelli, A., Tsiganis, K., Nesvorný, D., \& Gomes, R. 2011, AJ, 142, 152

Levy, J. S., Marchant, D. R., \& Head, J. W. 2010, Icarus, 206, 229

Malin, M. C., \& Zimbelman, J. R. 1986, Lunar Planet. Sci. XVII, 512

Mann, R. K., Jewitt, D., \& Lacerda, P. 2007, AJ, 134, 1133

Marchi, S., Barbieri, C., Kueppers, M., et al. 2010, P\&SS, 58, 1116

Marchi, S., Massironi, M., Vincent, J.-B., et al. 2012a, P\&SS, 66, 87

Marchi, S., McSween, H. Y., O'Brien, D. P., et al. 2012b, Science, 336, 690

Marchi, S., Bottke, W. F., Cohen, B. A., et al. 2013, Nature Geoscience, 6, 303

Marchi, S., Rickman, H., Massironi, M., et al. 2015, The geomorphology of Comet 67P: implications for the past collisional evolution and Formation, Lunar and Planetary Science Conference, \#1532

Massironi, M., et al. 2015, Nature, submitted

Morbidelli, A., \& Rickman, H. 2015, A\&A, 583, A43

Morbidelli, A., Marchi, S., Bottke, W. F., \& Kring, D. A. 2012, Earth Planet. Sci. Lett., 355, 144

Nesvorný, D., Youdin, A. N., \& Richardson, D. C. 2010, AJ, 140, 785

Noll, K. S., Grundy, W. M., Chiang, E. I., Margot, J.-L., \& Kern, S. D. 2008, in The Solar System Beyond Neptune, eds. M. A. Barucci, H. Boehnhardt, D. P. Cruikshank, \& A. Morbidelli (Tucson: Univ. Arizona), 345

Prialnik, D., Benkhoff, J., \& Podolak, M. 2004, in Comets II, eds. M. C. Festou,

H. U. Keller, \& H. A. Weaver (Tucson: Univ. Arizona), 359

Scheeres, D. J. 2007, Icarus, 189, 370

Sheppard, S. S., \& Jewitt, D. 2004, AJ, 127, 3023

Sierks, H., Barbieri, C., Lamy, P. L., et al. 2015, Science, 347, 1044

Stern, S. A., \& Weissman, P. R. 2001, Nature, 409, 589

Thomas, P., Veverka, J., A'Hearn, M. F., et al. 2005, Space Sci. Rev., 117, 193

Thomas, N., Sierks, H., Barbieri, C., et al. 2015, Science, 347, 0440

Tsiganis, K., Gomes, R., Morbidelli, A., \& Levison, H. F. 2005, Nature, 435, 459

Yasui, M., \& Arakawa, M. 2009, J. Geophys. Res., 114, 9004

Vincent, J.-B., Bodewits, D., Besse, S., et al. 2015, Nature, 523, 63

Wahlberg Jansson, K., \& Johansen, A. 2014, A\&A, 570, A47

Weidenschilling, S. J. 2008, Phys. Script., 130, 014021

Wetherill, G. W. 1967, J. Geophys. Res., 72, 2429

Wünnemann, K., Collins, G. S., \& Melosh, H. J. 2006, Icarus, 180, 514 